\title{
Crossover of Microscopic Dynamics in Metallic Supercooled Liquid Observed by NMR
}

\author{
Lilong $\mathrm{Li}^{1}{ }^{1}$ Jan Schroers, ${ }^{2}$ and Yue $\mathrm{Wu}^{1, *}$ \\ ${ }^{1}$ Department of Physics and Astronomy and Curriculum in Applied and Materials Sciences, University of North Carolina, \\ Chapel Hill, North Carolina 27599-3255, USA \\ ${ }^{2}$ W. M. Keck Laboratory of Engineering Materials, California Institute of Technology, Pasadena, California 91125, USA
}

(Received 7 July 2003; published 29 December 2003)

\begin{abstract}
Nuclear magnetic resonance is used to characterize local atomic motions in the glassy and supercooled liquid states of the bulk metallic glass system $\mathrm{Pd}_{43} \mathrm{Ni}_{10} \mathrm{Cu}_{27} \mathrm{P}_{20}$. The temperature dependence of the Knight shift reveals that certain local atomic motion decreases rapidly below a crossover temperature $T_{c}$ down to the glass transition temperature $T_{\mathrm{g}}$. Above $T_{c}$ as well as below $T_{\mathrm{g}}$ the meansquared amplitude of local motions depends linearly on the temperature. The observed gradual transition below $T_{c}$ is inconsistent with heterogeneity effects. It reveals that qualitative changes of microscopic properties in the supercooled liquid take place at temperatures significantly above $T_{\mathrm{g}}$.
\end{abstract}

DOI: $10.1103 /$ PhysRevLett.91.265502

PACS numbers: $63.50 .+\mathrm{x}, 61.43 .-\mathrm{j}, 76.60 .-\mathrm{k}$

An important issue of the nature of glass transition is whether it is a manifestation of qualitative changes of the microscopic property above $T_{\mathrm{g}}$ or a hidden phase transition below $T_{\mathrm{g}}[1,2]$. Among various theories, the modecoupling theory (MCT) predicts a qualitative change in the microscopic dynamics in the supercooled liquid at a crossover temperature $T_{c}$ significantly above $T_{\mathrm{g}}$ [3]. In a liquid of densely packed atoms, an atom is temporarily trapped inside the cage formed by neighboring atoms and undergoes vibrations and rattling before escaping the cage $[3,4]$. Here, the time dependence of the position vector $\vec{r}_{i}(t)$ of a given atom $i$ can be described by $\vec{r}_{i}(t)=$ $\vec{R}_{i}(t)+\vec{u}_{i}(t)$ where the rapidly changing $\vec{u}_{i}(t)$ describes local motions with time average $\left\langle\vec{u}_{i}(t)\right\rangle=0$ and the slowly varying $\vec{R}_{i}(t)$ describes the transition of the atom from one cage to another [5]. Consistent with this picture, MCT predicts that $\phi(q, t)$, the correlation function of density fluctuations with wave number $q$, undergoes a two-step relaxation process, called fast $\beta$ relaxation and $\alpha$ relaxation [3,4]. An important prediction of MCT is the temperature $(T)$ dependence of $\vec{u}_{i}$ associated with the fast $\beta$ relaxation $[3,4]$. The effect of $\vec{u}_{i}$ contributes to an effective Debye-Waller factor $f_{q}=\exp (-2 W)$. MCT predicts that $f_{q}$ increases critically from $f_{q}=f_{q}^{c}$ above a crossover temperature $T_{c}>T_{\mathrm{g}}$ to $f_{q}=f_{q}^{c}+h_{q} \sqrt{\varepsilon}$ below $T_{c}$ where $\varepsilon=\left(T_{c}-T\right) / T_{c}$ and $h_{q}$ is an amplitude. Experimentally, the separation between the time scales of $\alpha$ and fast $\beta$ relaxations in the temperature range of interest is often insufficient for model-independent determination of $f_{q}$ by neutron scattering [6]. So far, measurements of the effects of $\vec{u}_{i}$ have not revealed the critical behavior of $f_{q}$ in metallic supercooled liquids [7-9]. A recent diffusion measurement [10], which measures the effect of $\vec{R}_{i}(t)$, showed changes of diffusion mechanisms in the supercooled liquid region of $\mathrm{Pd}_{43} \mathrm{Ni}_{10} \mathrm{Cu}_{27} \mathrm{P}_{20}$, one of the best metallic glass formers $[11,12]$. Here, we introduce a nuclear magnetic resonance (NMR) method for probing $\vec{u}_{i}$ in $\mathrm{Pd}_{43} \mathrm{Ni}_{10} \mathrm{Cu}_{27} \mathrm{P}_{20}$ using the average Knight shift. The result reveals clearly a crossover behavior of the microscopic property in the supercooled liquid at $T_{c}>T_{\mathrm{g}}$.

The preparation of high purity $\mathrm{Pd}_{43} \mathrm{Ni}_{10} \mathrm{Cu}_{27} \mathrm{P}_{20}$ bulk metallic glass is described in detail elsewhere $[11,13] . T_{\mathrm{g}}$ and the liquidus temperature $T_{\text {liq }}$ are $580 \mathrm{~K}$ and $870 \mathrm{~K}$, respectively, as measured by differential scanning calorimetry (DSC) at a scanning rate of $0.33 \mathrm{~K} / \mathrm{s}$. NMR measurements were carried out at $9.4 \mathrm{~T}$ using a pulsed NMR spectrometer and a homemade high-temperature NMR probe. For NMR measurements samples were vacuum sealed in quartz tubes along with a small amount of $\mathrm{B}_{2} \mathrm{O}_{3}$ flux for the reduction of nucleation [13].

Figure 1 shows the room temperature (RT) ${ }^{31} \mathrm{P}$ NMR spectra of the glassy $\mathrm{Pd}_{43} \mathrm{Ni}_{10} \mathrm{Cu}_{27} \mathrm{P}_{20}$ sample, obtained by water quenching from the melt, and a crystalline

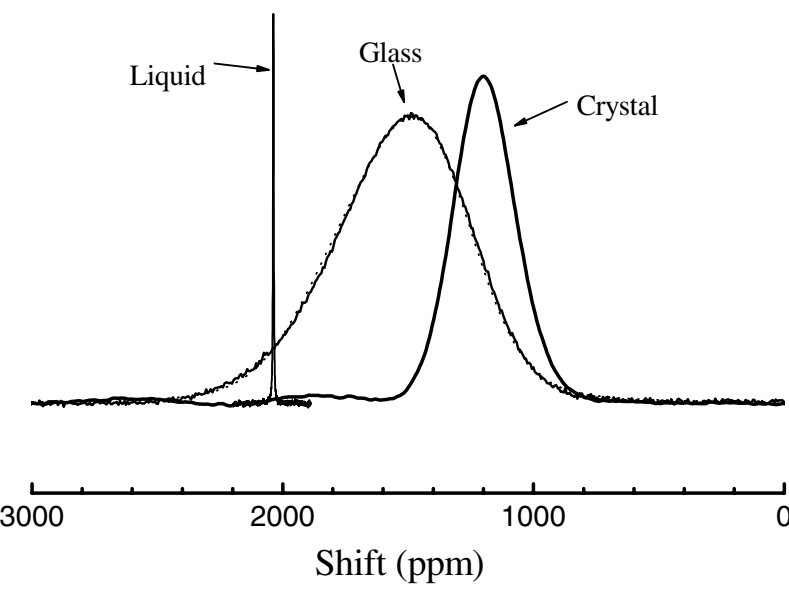

FIG. 1. ${ }^{31} \mathrm{P}$ NMR spectra of the glassy and crystallized samples at RT, detected using the Hahn echo sequence, and the spectrum of the liquid state taken at $1057 \mathrm{~K}$. The shift reference is ${ }^{31} \mathrm{P}$ in $85 \% \mathrm{H}_{3} \mathrm{PO}_{4}$. The dotted line is a powder pattern fit using a chemical shift anisotropy (CSA) parameter $\delta_{\text {aniso }}=370 \mathrm{ppm}, \eta=0$, and a $500 \mathrm{ppm}$ line broadening. 
sample obtained upon crystallization of the supercooled liquid at $767 \mathrm{~K} . \mathrm{A}^{31} \mathrm{P}$ spectrum of the liquid taken at $1057 \mathrm{~K}$ is also shown in Fig. 1. The RT spectrum of the glassy state is broad since the projected local magnetic field $B_{\text {loc }}$ along the external magnetic field direction varies with $\vec{R}_{i}$ from site to site. The ensemble average of $B_{\text {loc }}$ is represented by the average shift $K$ of the spectrum given by the first moment of the spectrum [14]. Figure 2 shows the $T$ dependence of $K$, which varies linearly both above $700 \mathrm{~K}$, with a slope of $0.75 \mathrm{ppm} / \mathrm{K}$, and below $T_{\mathrm{g}}$, with a slope of $0.27 \mathrm{ppm} / \mathrm{K}$. Data above $700 \mathrm{~K}$ were obtained by first heating the sample above $T_{\text {liq }}$ and then cooling directly to $T$ in situ for NMR measurement. The same results were obtained by cooling (or heating) directly from one $T<T_{\text {liq }}$ to another $T<T_{\text {liq }}$ (lower or higher) without going back to $T>T_{\text {liq }}$ in the absence of crystallization. Data below $700 \mathrm{~K}$ were obtained by heating the glassy sample from RT to the measurement temperature. The same results were obtained by direct in situ cooling from the melt to the measurement temperature. No time dependence of $K$ was observed at any temperature.

In metallic systems the dominant shift mechanism is the Knight shift. The main contribution $K_{\mathrm{s}}$ originates from the Fermi contact hyperfine interaction and can be expressed as [14,15]

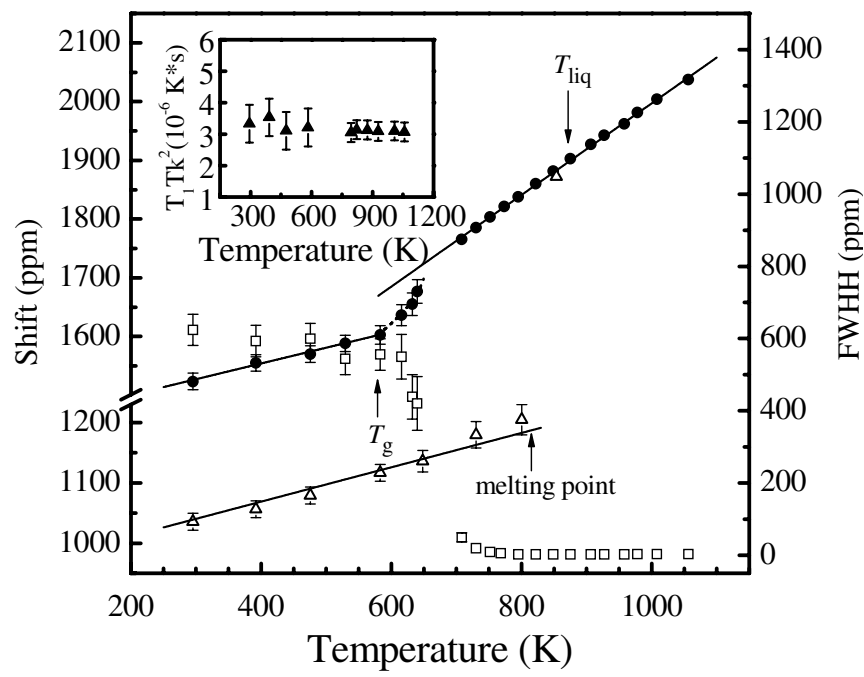

FIG. 2. The average shift $K$ measured at $9.4 \mathrm{~T}$ versus $T$ in the liquid, supercooled liquid, and glassy states (solid circle) as well as that of the crystallized sample (open triangle). The $T$ dependence of the ${ }^{31} \mathrm{P}$ spectral linewidth (full width at half height) is also shown (open square). The dashed line is a fit using Eq. (4) with $T_{c}=660 \mathrm{~K}$. The inset shows $T_{1} T K^{2}$ versus $T . T_{1}$ was measured using the saturation recovery method. The shift of the crystalline sample after melting is slightly lower than that of the supercooled liquid because homogeneous composition was restored only after heating above $T_{\text {liq }}$. Errors are comparable to or smaller than the sizes of symbols where error bars are not shown explicitly.

$$
K_{\mathrm{s}}=\frac{8 \pi}{3}\left\langle|\psi(0)|^{2}\right\rangle_{E_{\mathrm{F}}} \Omega \chi_{\text {Pauli }}
$$

where $\left\langle|\psi(0)|^{2}\right\rangle_{E_{\mathrm{F}}}$ is the density of the electron wave function at the nucleus averaged over all states at the Fermi level $E_{\mathrm{F}}$ and is normalized over an arbitrarily chosen volume $\Omega$. $\chi_{\text {Pauli }}$ is the Pauli paramagnetic susceptibility per unit volume. Under noninteracting electron approximation $\chi_{\text {Pauli }}$ is given by $\chi_{\text {Pauli }}=2 \mu_{\mathrm{B}}^{2} g\left(E_{\mathrm{F}}\right)$, where $\mu_{\mathrm{B}}$ is the Bohr magneton and $g\left(E_{\mathrm{F}}\right)$ is the density of states per unit volume at the Fermi level (for a single spin orientation). The Fermi contact hyperfine interaction also contributes to the nuclear spin-lattice relaxation rate $1 / T_{1}$ given by $[14,15]$

$$
T_{1}^{-1}=\frac{64}{9} \pi^{3} \hbar^{3} \gamma_{\mathrm{e}}^{2} \gamma_{\mathrm{n}}^{2}\left\langle|\psi(0)|^{2}\right\rangle_{E_{\mathrm{F}}}^{2} g^{2}\left(E_{\mathrm{F}}\right) k_{\mathrm{B}} T,
$$

where $\gamma_{\mathrm{e}}=2 \mu_{\mathrm{B}} / \hbar=1.76 \times 10^{11} \mathrm{~Hz} / \mathrm{T}$ is the electron gyromagnetic ratio, $\gamma_{\mathrm{n}}=1.08 \times 10^{8} \mathrm{~Hz} / \mathrm{T}$ is the gyromagnetic ratio of the ${ }^{31} \mathrm{P}$ nucleus, and $h=2 \pi \hbar$ and $k_{\mathrm{B}}$ are the Planck and Boltzmann constants, respectively. Equations (1) and (2) lead to the Korringa relation [14,15]

$$
T_{1} T K_{\mathrm{s}}^{2}=\frac{\hbar \gamma_{\mathrm{e}}^{2}}{4 \pi k_{\mathrm{B}} \gamma_{\mathrm{n}}^{2}} f=1.6 \times 10^{-6} f,
$$

where $f=1$ for noninteracting electrons. The inset in Fig. 2 shows the measured $T_{1} T K^{2}$. The measured $T_{1}$ is the same at 9.4 and $4.7 \mathrm{~T}$. As expected from Eq. (3), $T_{1} T K^{2}$ remains constant at a value of $3.4 \times 10^{-6}$ despite the large $T$ dependence of $K$. The measured value of $f=$ 2.1 implies a certain degree of electron-electron interactions typically found in metals. The constant $T_{1} T K^{2}$ and the value of $f=2.1$ are consistent with the conclusion of a previous NMR study in similar systems where $d$-band contributions to $T_{1}$ and $K$ are ruled out [16]. Thus, the $T$ dependence of $K$ cannot be attributed to the $d$-band effect [15].

A distribution of $K_{\mathrm{s}}$ caused by the distribution of local structures cannot be the single dominant factor for the linewidth of the RT spectrum. The high-frequency side of the spectrum would relax much faster than the lowfrequency side of the spectrum according to the Korringa relation if $K_{\mathrm{s}}$ variation dominates the linewidth. Observation shows that the relaxation rate is indistinguishable across the entire spectrum. The asymmetric line shape of the RT spectrum is characteristic of the powder pattern of anisotropic shift mechanisms such as the CSA. A fit using a CSA parameter $\delta_{\text {aniso }}=370 \mathrm{ppm}$ and an asymmetry parameter $\eta=0$ is shown in Fig. 1. Such a CSA value is typical for ${ }^{31} \mathrm{P}$ spectra $[17,18]$. In the presence of motions, the linewidth is determined by the distribution of the time-averaged $B_{\text {loc }}$ experienced by each ${ }^{31} \mathrm{P}$ spin over the duration of the time-domain NMR signal (about $9 \mu \mathrm{s}$ ). Rapid changes of $\vec{R}_{i}(t)$ over this time scale will result in all spins experiencing the 
same time-averaged $B_{\text {loc }}$ leading to motional line narrowing. This effect is expected to become significant above $T_{\mathrm{g}}$. Figure 2 shows that noticeable line narrowing occurs above $620 \mathrm{~K}$ reaching complete narrowing above $750 \mathrm{~K}$. However, $\vec{R}_{i}(t)$ does not lead to a different ensemble of local environments in the glassy and supercooled liquid states. The time-averaged $B_{\text {loc }}$ caused by $\vec{R}_{i}(t)$ is the same as the spatially averaged $B_{\text {loc }}$ obtained by the first moment calculation. Thus, diffusion does not lead to the $T$ dependence of $K$. In contrast, $\vec{u}_{i}(t)$ cannot lead directly to an efficient motional narrowing since it cannot cause a given ${ }^{31} \mathrm{P}$ spin to experience $B_{\text {loc }}$ at different phosphorus sites. The local averaging effect caused by $\vec{u}_{i}(t)$ could lead to small line narrowing, which might explain the observed weak $T$ dependence of the linewidth below $620 \mathrm{~K}$. Changes of $\vec{u}_{i}$ with $T$ do alter the ensemble of local fields. For instance, the closest possible atomic distance, which affects $B_{\text {loc }}$, will change with the increase of the amplitude of $\vec{u}_{i}(t)$. Therefore, the $T$ dependence of $\vec{u}_{i}$ could lead to the $T$ dependence of $K$ in both solid and liquid metals [19-22]. The electron wave function $\psi(\vec{r})$ in Eq. (1) can be represented formally in the form of tight binding as $\psi(\vec{r})=\psi_{0}(\vec{r})+\sum \psi_{i}\left[\vec{r}-\left(\vec{R}_{i}+\vec{u}_{i}\right)\right]$, where $\psi_{0}(\vec{r})$ represents orbitals of the atom at the origin associated with the observing nuclear spin and $\psi_{i}\left[\vec{r}-\left(\vec{R}_{i}+\right.\right.$ $\left.\vec{u}_{i}\right)$ ] represents orbitals of the neighboring atom at $\vec{r}_{i}(t)=$ $\vec{R}_{i}(t)+\vec{u}_{i}(t)$. Using Taylor expansion with respect to $\vec{u}_{i}$, it can be shown that the time average of $\left\langle|\psi(0)|^{2}\right\rangle_{E_{\mathrm{F}}}$ can be expressed as $\left\langle|\psi(0)|^{2}\right\rangle_{E_{\mathrm{F}}}=a_{0}+a_{1}\left\langle\vec{u}^{2}\right\rangle$ if higher order terms of $\vec{u}$ are negligible. Here, $a_{0}$ and $a_{1}$ are constants and $\vec{u}$ is an average of $\vec{u}_{i}$ 's of neighboring atoms. Other terms in Eq. (1) might also depend slightly on $\vec{u}$. Nevertheless, the lowest order term of $\vec{u}$ in $K$ is still proportional to $\left\langle\vec{u}^{2}\right\rangle$ because $\langle\vec{u}(t)\rangle=0$. It is important to realize that, unlike the linewidth, $K$ does not just probe motions within a certain time scale window. $K$ represents the average $B_{\text {loc }}$ over all time scales including the static effect, namely, the distribution of local environments. This makes $K$ particularly useful for detecting the onset of motions regardless of the range of time scales.

The linear $T$ dependence of the shift below $580 \mathrm{~K}$ shown in Fig. 2 suggests that $\left\langle\vec{u}^{2}\right\rangle \propto k_{\mathrm{B}} T$ as expected from the equipartition theorem for harmonic vibrations. The linear $T$ dependence of $K$ in the crystalline sample exhibits the same slope as the glassy state. This demonstrates that the ${ }^{31} \mathrm{P}$ Knight shift in this type of alloy is sensitive to atomic vibrations. It is expected that $K$ will become more sensitive to $T$ above $T_{\mathrm{g}}$ since sound velocities and elastic moduli in metallic glasses decrease above $T_{\mathrm{g}}[23,24]$. The observed linear $T$ dependence of $K$ with a larger slope above $700 \mathrm{~K}$ indeed agrees with this expectation. Also, an estimation based on $\Delta K_{\mathrm{s}} / K_{\mathrm{s}}=2 \Delta V / 3 V$ shows that the influence of the volume expansion $\Delta V / V$ of $1.2 \%$ from RT to $580 \mathrm{~K}$ and $3.6 \%$ from 580 to $1000 \mathrm{~K}$ [25] is negligible for the observed change of $K$. The large positive offset from the observed $K$ at $T_{\mathrm{g}}$ to the extrapo- lated value based on the data above $700 \mathrm{~K}$ (see Fig. 2) is inconsistent with heterogeneity-related gradual evolution from the solidlike behavior below $580 \mathrm{~K}$ to the liquidlike behavior above $700 \mathrm{~K}$. It indicates that some $\vec{u}_{i}(t)$ associated atomic motion present in the liquidlike region decreases rapidly upon cooling below $700 \mathrm{~K}$. A candidate of such atomic motion is the fast $\beta$ process associated with cage rattling. Since NMR is a local probe, it is particularly sensitive to such atomic motion. In the ideal form of MCT $\sqrt{\left\langle u^{2}\right\rangle}$ below $T_{c}$ is given by [26]

$$
\sqrt{\left\langle u^{2}\right\rangle}=u_{c}\left[1-\frac{1}{2} a \sqrt{\left(T_{c}-T\right) / T_{c}}\right]
$$

where $a>0$ and $u_{c}$ is the magnitude of rattling above $T_{c}$. A fit of $K$ is shown in Fig. 2 using Eq. (4) with $T_{c}=$ $660 \pm 10 \mathrm{~K}$ assuming the shift is proportional to $\left\langle u^{2}\right\rangle$ over the entire temperature range. The $T_{c}$ value agrees within the range of uncertainties with diffusion results $[10,27]$. This by no means implies that the change is abrupt at $660 \mathrm{~K}$. The change might be gradual below $700 \mathrm{~K}$ and the effective $T_{c}$ might be as high as $700 \mathrm{~K}$. The detail is obscured by the lack of $K$ data in that temperature range due to fast crystallization. It is interesting to note that no difference in $K$ was observed at any temperature by changing the quenching rate of the sample as well as by annealing. Samples vitrified under different cooling rates of $10^{2}$ and $10^{4} \mathrm{~K} / \mathrm{s}$ give exactly the same shift. Furthermore, annealing of a sample, originally obtained by quenching at a cooling rate of $10^{2} \mathrm{~K} / \mathrm{s}$, for $700 \mathrm{~min}$ at $573 \mathrm{~K}$ in DSC does not cause any change of $K$. This shows that the observed shift in the glassy state does not depend on the residual structural relaxation with the employed quenching rate. A much faster quenching rate is needed to elucidate the role of local structural changes on the observed $T$ dependence of the shift.

Our NMR result reveals for the first time a qualitative change of local properties in a metallic supercooled liquid significantly above $T_{\mathrm{g}}$. It is shown that certain local atomic motion changes rapidly between a crossover temperature $T_{c}>T_{\mathrm{g}}$ and a temperature very close to $T_{\mathrm{g}}$ in the supercooled liquid region of $\mathrm{Pd}_{43} \mathrm{Ni}_{10} \mathrm{Cu}_{27} \mathrm{P}_{20}$. This gradual transition is inconsistent with the heterogeneity effect. Above $T_{c}$, the mean-squared amplitude of local motions depends linearly on $k_{\mathrm{B}} T$. This is also the case between $T_{\mathrm{g}}$ and RT. Further NMR studies of different nuclei and different systems as well as effects of a fast quenching rate will shed more light on the nature of the observed crossover behavior.

We thank Professor W. L. Johnson, Professor J. Hernandez, and Professor T. Egami for very helpful discussions. This work was supported by the U.S. Army Research Office (Grant No. DAAD19-02-10225) and by the National Science Foundation (Grant No. DMR-0139452). 
*To whom all correspondence should be addressed. Electronic address: yuewu@physics.unc.edu

[1] M. D. Ediger, C. A. Angell, and S. R. Nagel, J. Phys. Chem. 100, 13200 (1996).

[2] P. G. Debenedetti and F. H. Stillinger, Nature (London) 410, 259 (2001).

[3] W. Götze and L. Sjögren, Rep. Prog. Phys. 55, 241 (1992).

[4] E. Donth, The Glass Transition (Springer, Berlin, 2001).

[5] F. Mezei, in Liquids, Freezing and Glass Transition, edited by J. P. Hansen, D. Levesque, and J. Zinn-Justin (Elsevier, Amsterdam, 1991), p. 631.

[6] F. Mezei and M. Russina, J. Phys. Condens. Matter 11, A341 (1999).

[7] A. Meyer, J. Wuttke, W. Petry, A. Peker, R. Bormann, G. Coddens, L. Kranich, O. G. Randl, and H. Schober, Phys. Rev. B 53, 12107 (1996).

[8] A. Meyer, H. Franz, B. Sepiol, J. Wuttke, and W. Petry, Europhys. Lett. 36, 379 (1996).

[9] A. Meyer, J. Wuttke, W. Petry, O. G. Randl, and H. Schober, Phys. Rev. Lett. 80, 4454 (1998).

[10] V. Zöllmer, K. Rätzke, F. Faupel, and A. Meyer, Phys. Rev. Lett. 90, 195502 (2003).

[11] I.-R. Lu, G. Wilde, G. P. Görler, and R. Willnecker, J. NonCryst. Solids 250-252, 577 (1999).

[12] N. Nishiyama and A. Inoue, Acta Mater. 47, 1487 (1999).
[13] J. Schroers, Y. Wu, and W. L. Johnson, Philos. Mag. A 82, 1207 (2002).

[14] A. Abragam, Principles of Nuclear Magnetism (Clarendon Press, Oxford, 1961).

[15] J. Winter, Magnetic Resonance in Metals (Oxford, New York, 1971).

[16] W. A. Hines, K. Glover, W. G. Clark, L. T. Kabacoff, C. U. Modzelewski, R. Hasegawa, and P. Duwez, Phys. Rev. B 21, 3771 (1980).

[17] T. M. Alam and R. K. Brow, J. Non-Cryst. Solids 223, 1 (1998).

[18] S. T. Oyama et al., J. Phys. Chem. B 106, 1913 (2002).

[19] R. V. Kasowski and L. Falicov, Phys. Rev. Lett. 22, 1001 (1969).

[20] R.V. Kasowski, Phys. Rev. 187, 891 (1969).

[21] A. L. Ritter and J. A. Gardner, Phys. Rev. B 3, 46 (1971).

[22] G. B. Benedek and T. Kushida, J. Phys. Chem. Solids 5, 241 (1958).

[23] N. Nishiyama, A. Inoue, and J. Z. Jiang, Appl. Phys. Lett. 78, 1985 (2001).

[24] J. M. Pelletier, B. Van de Moortèle, I. R. Lu, Mater. Sci. Eng., A 336, 190 (2002).

[25] I.-R. Lu, G. P. Görler, and R. Willnecker, Appl. Phys. Lett. 80, 4534 (2002).

[26] W. Götze, Phys. Scr. 34, 66 (1986).

[27] A. Meyer, R. Busch, and H. Schober, Phys. Rev. Lett. 83, 5027 (1999). 